\title{
The Zeros of a Certain Homogeneous Difference Polynomials of Meromorphic Functions*
}

\author{
Qian Lu' ${ }^{1}$, Qilong Liao \\ ${ }^{1}$ Department of Mathematics, Southwest University of Science and Technology, Mianyang, China \\ ${ }^{2}$ Department of Material Science and Engineer, Southwest University of Science and Technology, Mianyang, China \\ Email: luqiankuo1965@hotmail.com, liaoql@swust.edu.cn
}

Received October 17, 2012; revised November 26, 2012; accepted December 4, 2012

\begin{abstract}
Let $f(z)$ be a function transcendental and meromorphic in the plane of growth order less than 1 . This paper focuses on discussing and estimating the number of the zeros of a certain homogeneous difference polynomials of degree $k$ in $f(z)$, and obtains that this certain homogeneous difference polynomials has infinitely many zeros.
\end{abstract}

Keywords: Meromorphic Functions; Zeros; Homogeneous Difference Polynomials

\section{Introduction and the Main Result}

Let $f(z)$ be a function transcendental and meromorphic in the plane. In what follow, we denote the convergence exponent of the zeros of $f(z)$ by $\lambda(f)$, the growth order of $f(z)$ by $\sigma(f)$, and the lower order of $f(z)$ by $\mu(f)$.

Following Whittaker [1], define the forward differences to be $k$ times iteration $\Delta^{k}$ of the difference operator $\Delta$, that is,

$$
\begin{aligned}
& \Delta f(z)=f(z+1)-f(z), \\
& \Delta^{k} f(z)=\Delta^{k-1} f(z+1)-\Delta^{k-1} f(z) .
\end{aligned}
$$

Recently, a number of papers research on complex difference equations and differences analogues of Nevanlinna's theory [2-6]. Bergweiler and Langley [7] firstly investigated the existence of zeros of $\Delta f(z)$, and obtained a result as follow.

Theorem 1.1. Let $f$ be a function transcendental and meromorphic of lower order $\mu(f)<\mu<1$ in the plane. Let $c \in C \backslash\{0\}$ be such that at most finitely many poles $z_{j}, z_{k}$ of $f(z)$ satisfy $z_{j}-z_{k}=c$. Then $g(z)=f(z+c)-f(z)$ has infinitely many zeros.

In 2008, Z. X. Chen and K. H. Shon [8].

Theorem 1.2. Let $n \in N$ and $f$ be a function transcendental and meromorphic of lower order $\mu(f)<\mu<1$ in the plane. Let $c \in C \backslash\{0\}$ and a set $B=\{b j\}$ consist of all poles of $f(z)$, such that

$$
b_{j}+k c \notin B(k=1,2, \cdots, n)
$$

*Supported by China Industrial Technology Development Program (B3120110001) and Sichuan Provincial Science and Technology Department of Applied Foundation Project (07JY029-006). at most except finitely many exceptions. Then $\Delta^{n} f(z)$ has infinitely many zeros.

In 2009, Z. X. Chen and K. H. Shon [9] continue to investigate the existence of the zeros of the difference polynomials defined as follows

$$
\begin{aligned}
& g(z)=f\left(z+c_{1}\right)+f\left(z+c_{2}\right)-2 f(z) \\
& g_{2}(z)=f\left(z+c_{1}\right) \cdot f\left(z+c_{2}\right)-f^{2}(z)
\end{aligned}
$$

and obtained two results.

Theorem 1.3. Let $f$ be a function transcendental and meromorphic of growth order $\sigma(f)=\sigma<1$, and $c_{1}, c_{2}$ be two complex numbers, such that $c_{1}, c_{2} \in C \backslash\{0\}$, and $c_{1}+c_{2} \neq 0$. If $f(z)$ has at most finitely many poles $p_{j}, p_{s}$ satisfying $p_{j}-p_{s}=k_{1} c_{1}+k_{2} c_{2}$

$\left(k_{d}=0, \pm 1, d=1,2\right)$, then $g(z)$ has infinitely many zeros, and $\lambda(g)=\sigma(g)=\sigma$.

In particular, if $f(z)$ has at most finitely many zeros $z_{j}$ satisfying $f\left(z_{j}+c_{1}\right)+f\left(z_{j}+c_{2}\right)=0$, then $G(z)=g(z) / f(z)$ has also infinitely many zeros, and $\lambda(G)=\sigma(G)=\sigma$.

Theorem 1.4. Let $f(z), c_{1}, c_{2}$ satisfy the conditions in Theorem 1.3, If $f(z)$ has at most finitely many poles $b_{j}$ satisfying

$$
f\left(b_{j}+k_{1} c_{1}+k_{2} c_{2}\right)=0, \infty\left(k_{d}=0, \pm 1, d=1,2\right),
$$

then $g_{2}(z)$ has infinitely many zeros, and $\lambda\left(g_{2}\right)=\sigma\left(g_{2}\right)=\sigma$.

In particular, if $f(z)$ has at most finitely many zeros $z_{j}, Z_{s}$ such that $Z_{j}-z_{s}=c_{1}, c_{2}$, then

$G_{2}(z)=g_{2}(z) / f^{2}(z)$ has also infinitely many zeros, and 


$$
\lambda\left(G_{2}\right)=\sigma\left(G_{2}\right)=\sigma .
$$

It is not difficult to understand that $g(z)$ defined by (1.2) is more general difference polynomials than $\Delta f(z)$ or $\Delta^{2} f(z)$ and Theorem 1.3 extends Theorem 1.1. Therefore, we pose naturally one question whether more general difference polynomials than $g_{2}(z)$ defined by (1.3) has also infinitely many zeros. In this paper, we focus on research a certain homogeneous difference polynomials and affirm to answer this problem.

Theorem 1.5. Suppose that $k$ is a positive integer, $k \geq 1$. Let $f(z)$ be a function transcendental and meromorphic of growth order $\sigma(f)=\sigma<1$, and there exists $k$ complex numbers $c_{j} \in C \backslash\{0\} j=1,2, \cdots, k$ such that $\sum_{j=1}^{k} c_{j} \neq 0$. If $f(z)$ has at most finitely many poles $b_{j}$ satisfying

$$
\begin{aligned}
& f\left(b_{j}+l_{1} c_{1}+l_{2} c_{2}+\cdots+l_{k} c_{k}\right)=0, \infty, \\
& l_{d}=0, \pm 1, d=1,2, \cdots, k .
\end{aligned}
$$

Then $H_{k}(f)=\prod_{i=1}^{k} f\left(z+c_{i}\right)-f^{k}(z)$ has infinitely many zeros, and $\lambda\left(H_{k}\right)=\sigma\left(H_{k}\right)=\sigma$.

In particular, if $f(z)$ has at most finitely many zeros $z_{j}, z_{s}$ satisfying $z_{j}-z_{s}=c_{1}, c_{2}, \cdots, c_{k}$, then

$\psi_{k}(f)=H_{k}(f) / f^{k}(z)$ has also infinitely many zeros, and $\lambda\left(\psi_{k}\right)=\sigma\left(\psi_{k}\right)=\sigma$.

\section{Lemmas}

Lemma 2.1. (see [7]) Let $f$ be a function transcendental and meromorphic in the plane of growth order less than 1 , and $h>0$. Then there exists an $\varepsilon$-set $E$ such that

$$
f(z+c)-f(z)=c f^{\prime}(z)(1+o(1)),
$$

as $z \rightarrow \infty, z \in C \backslash E$, uniformly in $c$ for $|c| \leq h$.

Lemma 2.2. (see [7]) Let $f(z)$ be a function transcendental and meromorphic in the plane of lower order $\mu(f)=\mu<1$. Then there exists arbitrarily large $R$ with the following properties. First,

$$
T\left(32 R, f^{\prime}\right)<R^{\mu} .
$$

Second, there exists a set $J_{R} \subseteq[R / 2, R]$ of linear measure $m\left(J_{R}\right)=\int_{J_{R}} \frac{\mathrm{d} r}{R-r}=[1-o(1)] R / 2$, such that for $r \in J_{R}$,

$$
f(z+1)-f(z) \sim f^{\prime}(z)
$$

on $|z|=r$.

Lemma 2.3. Let $f(z)$ be a function transcendental and meromorphic in the plane with growth order $\sigma(f)=\sigma<1$. Supposed that $\sum_{j=1}^{k} c_{j} \neq 0$. If the homoge- neous difference polynomials

$$
H_{k}(f)=\prod_{j=1}^{k} f\left(z+c_{j}\right)-f^{k}(z),
$$

or quotient of difference polynomials

$$
\psi_{k}(f)=H_{k}(f) / f^{k}(z)
$$

is rational functions, then $f(z)$ has at most finite many poles.

Proof. Without loss of generality, we assume that $c_{1}=$ 1 . Because that the homogeneous difference polynomials $H_{k}(f)$ is rational, there exists a rational functions $R(z)$ such that

$$
H_{k}(f)=\prod_{j=1}^{k} f\left(z+c_{j}\right)-f^{k}(z)=R(z) .
$$

Set

$$
B=\{b j=x j+i y j \mid R(b j)=\infty, j=1,2, \cdots, s\},
$$

and

$$
M=\max \left\{|x j|+|y j|+1+\sum_{j=1}^{k}\left|c_{j}\right|: 1 \leq j \leq s\right\} .
$$

So there exists no poles of $R(z)$ in the domain

$$
\begin{aligned}
& D_{1}=\{z: \operatorname{Re} z>M\}, \\
& D_{2}=\{z: \operatorname{Re} z<-M\}, \\
& D_{3}=\{z: \operatorname{Im} z>M\}
\end{aligned}
$$

and

$$
D_{4}=\{z: \operatorname{Im} z<-M\} .
$$

Now we complete the proof of the conclusion that $f(z)$ has at most finite.

Now we complete the proof of the conclusion that $f(z)$ has at most finite many poles. Suppose not, there exists one domain $D_{j}$, for example $D_{1}$, in which $f(z)$ has infinitely many poles. We assume that the set $A=\left\{z_{j}\right\}$ consists of all poles of $f(z)$ in $D_{1}$ and $M<\left|z_{1}\right| \leq\left|z_{2}\right| \leq \cdots$ and divide it into two cases:

Case 1. There exists $z_{d} \in A$, such that for an arbitrary $b_{j} \in B$, there does not exist $m_{1}, m_{2}, \cdots, m_{k} \in N$ such that $b_{j}=z_{d}+m_{1}+\sum_{r=2}^{k} m_{r} c_{r}$, that is, for an arbitrary $m_{1}, m_{2}, \cdots, m_{k} \in N$, we have $b_{j} \neq z_{d}+m_{1}+\sum_{r=2}^{k} m_{r} c_{r}$. In fact, since $\operatorname{Re} b_{j}<M$ and $\operatorname{Re} z_{d}>M$, this case appears whenever $\operatorname{Re} c_{j}>0$ for every $i=2,3, \cdots, k$. Therefore, we know $R\left(z_{d}+m_{1}+\sum_{r=2}^{k} m_{r} c_{r}\right) \neq \infty$ and that there exists a unbounded subsequence set $A_{1}=\left\{z_{d}+m_{1}+m_{2} c_{2}+\cdots+m_{k} c_{k}\right\}$, in which every 
$z_{d}+m_{1}+\sum_{r=2}^{k} m_{r} c_{r}$ is the poles of $f(z)$. Hence we know that there are at least one in these signs $m_{1}, m_{2}, \cdots, m_{k}$, which takes every positive integer, for instance, $m_{1}$ takes every positive integer.

Thus, $\lambda(f)=1$, which contradicts the hypothesis of Lemma 2.3.

Case 2. There exists $b_{0} \in B$, such that for every $z_{j} \in A$, there exists $m_{j 1}, m_{j 2}, \cdots, m_{j k} \in N$, such that $b_{0}=z_{j}+m_{j 1}+m_{j 2} c_{2}+\cdots+m_{j k} c_{k}$. From $\operatorname{Re}\left(z_{j}\right)>M$ and $\operatorname{Re}\left(b_{0}\right)<M$, we have that $\sum_{r=2}^{k} m_{j r} \operatorname{Re} c_{r}<0$. As the set $A$ is infinite and $B$ has only a finite elementary, there exists $b_{0} \in B$, satisfying

$$
b_{0}=z_{1}+m_{11}+\sum_{r=2}^{k} m_{1 r} c_{r}=z_{2}+m_{21}+\sum_{r=2}^{k} m_{2 r} c_{r}=\cdots
$$

By putting $\left\{z_{j}+m_{j 1}+\sum_{r=2}^{k} m_{j r} c_{r}\right\}$ in order again, we have the following express

$$
m_{j l} \leq m_{j+1, l}, l=1,2, \cdots, k ; j=1,2, \cdots,
$$

and

$$
\begin{aligned}
& z_{j+1}=z_{j}+\left(m_{j 1}-m_{j+1,1}\right)+\sum_{r=2}^{k}\left(m_{j r}-m_{j+1, r}\right) c_{r}, \\
& j=1,2, \cdots,
\end{aligned}
$$

where

$$
0 \geq m_{1 r}-m_{j r} \geq m_{1 r}-m_{j+1, r}, r=1,2, \cdots, k ; j=1,2, \cdots, .
$$

Now set

$$
\begin{aligned}
z_{3 i j \cdots s}= & z_{1}+\left(m_{11}-m_{31}+i\right)+\left(m_{12}-m_{32}+j\right) c_{2}+\cdots \\
& +\left(m_{1 k}-m_{3 k}+s\right) c_{k},
\end{aligned}
$$

where

$$
\begin{gathered}
i=0,1,2, \cdots,\left(m_{11}-m_{21}\right)-\left(m_{11}-m_{31}\right) \\
j=0,1,2, \cdots,\left(m_{12}-m_{22}\right)-\left(m_{12}-m_{32}\right) \\
\vdots \\
s=0,1,2, \cdots,\left(m_{1 k}-m_{2 k}\right)-\left(m_{1 k}-m_{3 k}\right) .
\end{gathered}
$$

Since $\operatorname{Re}\left(z_{3 i j \ldots s}\right)$ are between $\operatorname{Re}\left(z_{2}\right)$ and $\operatorname{Re}\left(z_{3}\right)$, $\operatorname{Im}\left(z_{3 i j \ldots s}\right)$ are between $\operatorname{Im}\left(z_{2}\right)$ and $\operatorname{Im}\left(z_{3}\right)$, we know that $z_{3 i j \cdots s} \in D_{1}$, that is, $R\left(z_{3 i j \cdots s}\right) \neq \infty$. From $f\left(z_{3}\right)=\infty, R\left(z_{3 i j \cdots s}\right) \neq \infty$, and (2.4), we know that one of $z_{310 \cdots 0}, z_{301 \cdots 0}, \cdots$, and $z_{310 \cdots 0}$ is the pole of $f(z)$. If $z_{310 \cdots 0}$ is the pole of $f(z)$, then from the some argument above we have one of $z_{320 \cdots 0}, z_{311 \cdots 0}, \cdots$, and $z_{310 \cdots 1}$ is also the pole of $f(z)$. If $z_{301 \cdots 0}$ is the pole of $f(z)$, then one of $z_{311 \cdots 0}, z_{302 \cdots 0}, \cdots$, and $z_{301 \cdots 1}$ is also the pole of $f(z)$. On the analogy of this, it is not difficult to find there exists at least one of $i, j, \cdots, s$, for instance, we assume that is $j$, such that $j$ takes all value of $0,1,2, \cdots,\left(m_{12}-m_{22}\right)-\left(m_{12}-m_{32}\right)$. From $z_{4}$ to $z_{3}, z_{5}$ to $Z_{4}$, and $Z_{n}$ to $Z_{n-1}$, repeating above proceeding, we have

$$
\begin{aligned}
z_{n i j \cdots s}= & z_{n-1}+\left(m_{n-1,1}-m_{n 1}+i\right)+\left(m_{n-1, r}-m_{n r}\right) c_{r}+\cdots \\
& +\left(m_{n-1 k}-m_{n k}\right) c_{k}, n=2,3, \cdots
\end{aligned}
$$

where

$$
\begin{gathered}
i=0,1,2, \cdots, m_{n-1,1}-m_{n 1} \\
j=0,1,2, \cdots, m_{n-1,2}-m_{n 2} \\
\vdots \\
s=0,1,2, \cdots, m_{n-1, k}-m_{n k} .
\end{gathered}
$$

Therefore, we can see that there exist infinite many poles of $f(z)$ whose expressions are as follows

$$
\begin{aligned}
z_{n i j \cdots s}= & z_{1}+\left(m_{11}-m_{n 1}+i\right)+\left(m_{1 r}-m_{n r}+j\right) c_{r}+\cdots \\
& +\left(m_{1 k}-m_{n k}+s\right) c_{k}, n=2,3, \cdots,
\end{aligned}
$$

where

$$
\begin{gathered}
i=0,1,2, \cdots, m_{n, 1}-m_{11} \\
j=0,1,2, \cdots, m_{n, 2}-m_{12} \\
\vdots \\
s=0,1,2, \cdots, m_{n, k}-m_{1 k} .
\end{gathered}
$$

in which we can find that one of $i, j, \cdots, s$ takes every positive integer. Thus, $\lambda(f)$, which still contradict the hypothesis on the growth order of $f(z)$ in Lemma 2.3.

By the similar method to above, it is easy to prove that $f(z)$ has at most finite many poles whenever quotient of difference polynomials

$$
\psi_{k}(f)=H_{k}(f) / f^{k}(z)
$$

is rational functions.

Lemma 2.4. Let $f(z)$ be a function transcendental and meromorphic in the plane with growth order $\sigma(f)=\sigma<1$. Supposed that $\sum_{j=1}^{k} c_{j} \neq 0$, then the homogeneous difference polynomials

$$
H_{k}(f)=\prod_{j=1}^{k} f\left(z+c_{j}\right)-f^{k}(z)
$$

and

$$
H_{k}(f) / f^{k}(z)
$$

also are transcendental.

Proof. Suppose first that there exists a rational function $R(z)$, such that

$$
H_{k}(f)=\prod_{j=1}^{k} f\left(z+c_{j}\right)-f^{k}(z)=R(z)
$$

By Lemma 2.3, $f(z)$ has at most finite many poles. 
Again from Lemma 2.1, there exists $\varepsilon-\operatorname{set} E$ such that as $z \rightarrow \infty(z \in C \backslash E)$, we have

$$
f\left(z+c_{j}\right)-f(z)=c_{j} f^{\prime}(z)(1+o(1)), j=1,2, \cdots, k
$$

It follows that from (2.6) and (2.7)

$$
\begin{aligned}
& f^{\prime}(z)\left\{c_{1} c_{2} \cdots c_{k}\left(f^{\prime}(z)\right)^{k-1}(1+o(1))\right. \\
& +\cdots+\sum_{1 \leq j_{1} \leq \cdots \leq j_{m} \leq k} c_{j_{1}} c_{j_{2}} \cdots c_{j_{m}}\left(f^{\prime}(z)\right)^{m-1}(f(z))^{k-m}(1+o(1)) \\
& \left.+\cdots+\sum_{1 \leq j \leq k} c_{j}(f(z))^{k-1}(1+o(1))\right\}=R(z)
\end{aligned}
$$

We write $d(z)$ for a polynomial formed by the pole of $f(z)$, and $f_{0}(z)=f(z) d(z)$. So $f_{0}(z)$ is an entire function, and $\sigma\left(f_{0}\right)=\sigma(f)=\sigma<1$. With the standard result in the Wiman-Valiron Theory, we know that there exists a subset $F \subset(1,+\infty)$ with finite logarithmic measure $\int_{F} \frac{\mathrm{d} r}{r}<+\infty$, in which for an sufficiently large $r \notin F,\left|f_{0}(z)\right|=M\left(r, f_{0}\right),|z|=r$, the following equality holds

$$
\frac{f_{0}^{\prime}(z)}{f_{0}(z)}=\frac{v(r)}{z}(1+o(1))
$$

Thus,

$$
\frac{f^{\prime}(z)}{f(z)}=\frac{f_{0}^{\prime}(z)}{f_{0}(z)}-\frac{d^{\prime}(z)}{d(z)}=\frac{v(r)}{z}(1+o(1))
$$

where $v(r) / z \rightarrow \infty$, and $v(r) \rightarrow \infty$, as $z \rightarrow \infty$. Set $F_{1}=\{|z|: z \in E\}$. Since $E$ is $\varepsilon$-set, we have that $F_{1}$ also is of finite logarithmic measure. Therefore, for all $z$, $|z| \notin[0,1] \cup F \cup F_{1}$, and

$$
\left|f_{0}(z)\right|=M\left(r, f_{0}\right),
$$

we immediately deduce that from (2.8) and (2.9)

$$
\begin{aligned}
& \sum_{m=1}^{k} \sum_{1 \leq j_{1} \leq \cdots \leq j_{m} \leq k}\left(\prod_{t=1}^{m} c_{j_{t}}\right)\left(\frac{v(r)}{z}\right)^{m-1}(1+o(1)) \\
& =\frac{R(z) d^{k} z}{M^{k}\left(r, f_{0}\right)} \cdot \frac{1}{v(r)}(1+o(1))
\end{aligned}
$$

Since $\sigma\left(f_{0}\right)=\sigma<1$ and $f_{0}$ is transcendental, there exists a sequence $\mid r \notin[0,1] \cup F \cup F_{1} r_{n} \rightarrow \infty$, such that for arbitrary $\varepsilon>0$, we have that

$$
\begin{gathered}
\exp \left(k r_{n}^{\sigma-\varepsilon}\right)<M\left(r_{n}, f_{0}\right)^{k}<\exp \left(k r_{n}^{\sigma+\varepsilon}\right) \\
v(r) / z \rightarrow 0, v(r) \rightarrow \infty(z \rightarrow \infty) .
\end{gathered}
$$

Then, we induce that from (2.4) and (2.11)

$$
\frac{r_{n}^{\tau}(1+o(1))}{\exp \left(k r_{n}^{\sigma+\varepsilon}\right)}<\frac{\left|R(z) d(z)^{k} z\right|}{M\left(r_{n}, f_{0}\right)^{k}}<\frac{r_{n}^{\tau}(1+o(1))}{\exp \left(k r_{n}^{\sigma-\varepsilon}\right)}
$$

Therefore, from (2.12) and (2.13) we have

$$
\frac{R(z) d^{k} z}{M^{k}\left(r, f_{0}\right)} \rightarrow 0\left(r_{n} \rightarrow \infty\right)
$$

By (2.10), (2.12), and (2.14), we deduce easily that $c_{1}+c_{2}+\cdots+c_{k}=0$, which contradicts the assumption on $c_{1}+c_{2}+\cdots+c_{k} \neq 0$, that is, $H_{k}(f(z))$ transcendental.

Lemma 2.5. Let $f(z)$ be a function transcendental and meromorphic in the plane, whose growth orde $\sigma(f)=\sigma<1$. Supposed that $a_{1}, a_{2}, \cdots, a_{k} \in C \backslash\{0\}$, and $\bar{\lambda}(1 / f)=\lambda(1 / f)$. Then

$\max \left\{\lambda\left(f^{\prime}\right), \lambda\left(a_{1}\left(f^{\prime}\right)^{k-1}+a_{2}\left(f^{\prime}\right)^{k-2} f+\cdots+a_{k} f^{k-1}\right\}=\sigma\right.$

Proof. For $f(z)$ of growth order $\sigma(f)=\sigma<1$, from Hadamard's factorization theorem we have

$$
\begin{aligned}
& f(z)=p(z) q(z), \\
& f^{\prime}(z)=p_{1}(z) q_{1}(z)
\end{aligned}
$$

where $p(z)\left(p_{1}(z)\right)$ and $q(z)\left(q_{1}(z)\right)$ are respectively the canonical product of zeros and poles of $f(z)\left(f^{\prime}(z)\right)$, satisfying

$$
(p(z), q(z))=1\left(\left(p_{1}(z), q_{1}(z)\right)=1\right) .
$$

From (2.15), we have

$$
\begin{aligned}
\sigma\left(f^{\prime}\right) & =\max \left(\sigma\left(p_{1}(z)\right), \sigma\left(q_{1}(z)\right)\right) \\
& =\max \left(\lambda\left(f^{\prime}(z)\right), \lambda\left(1 / f^{\prime}(z)\right)\right)=\sigma .
\end{aligned}
$$

Therefore, if $\lambda\left(f^{\prime}\right)<\sigma$, we deduce that $\lambda\left(1 / f^{\prime}\right)=\sigma$. For $\bar{\lambda}(1 / f)=\lambda(1 / f)$, the following equations hold

$$
\lambda\left(1 / f^{\prime}\right)=\lambda(1 / f)=\bar{\lambda}(1 / f)=\lambda(f)=\sigma
$$

We have that from $\lambda\left(f^{\prime}\right)<\sigma$ and (2.16)

$$
\begin{aligned}
& \sigma(p)=\sigma\left(p_{1}\right)=\sigma(f), \\
& \sigma(q)=\sigma\left(q_{1}\right)<\sigma(f)
\end{aligned} .
$$

If $z_{0}$ is a poles of $f(z)$ with multiplicity $m$, then $z_{0}$ must be a poles of $f^{\prime}(z)$ with multiplicity $m+1$, so that we denote $q_{1}(z)$ by $q(z) d(z)$, that is,

$$
q_{1}(z)=q(z) d(z)
$$

where $d(z)$ is a canonical product of distinct poles of $d(z)$. By (2.16), we obtain that

$$
\sigma(d)=\lambda(d)=\bar{\lambda}(f)=\sigma(f)=\sigma .
$$

From (2.15) and (2.18), we deduce that 


$$
\begin{aligned}
& a_{1}\left(f^{\prime}\right)^{k-1}+a_{2}\left(f^{\prime}\right)^{k-2} f^{1}+\cdots+a_{k} f^{k-1} \\
& =\frac{a_{1}\left(p_{1}\right)^{k-1}+a_{2}\left(p_{1}\right)^{k-2}(p d)^{1}+\cdots+a_{k}(p d)^{k-1}}{q_{1}^{k-1}} .
\end{aligned}
$$

Thus, if $z_{0}$ is the pole of $f^{\prime}(z)$ (that is, $q_{1}\left(z_{0}\right)=0$ ), then $d\left(z_{0}\right)=0, p\left(z_{0}\right) \neq 0, \infty$, but $p_{1}\left(z_{0}\right) \neq 0, \infty$. Hence, we have that $z_{0}$ is not the zero of

$$
a_{1}\left(f^{\prime}\right)^{k-1}+a_{2}\left(f^{\prime}\right)^{k-2} f+\cdots+a_{k} f^{k-1} .
$$

So that

$$
\left(a_{1}\left(p_{1}\right)^{k-1}+a_{2}\left(p_{1}\right)^{k-2}(p d)^{1}+\cdots+a_{k}(p d)^{k-1}, q_{1}^{k-1}\right)=1,
$$

and

$$
\begin{aligned}
& \lambda\left(a_{1}\left(f^{\prime}\right)^{k-1}+a_{2}\left(f^{\prime}\right)^{k-2} f+\cdots+a_{k} f^{k-1}\right) \\
& =\lambda\left(a_{1}\left(p_{1}\right)^{k-1}+a_{2}\left(p_{1}\right)^{k-2}(p d)^{1}+\cdots+a_{k}(p d)^{k-1}\right) \\
& =\sigma\left(a_{1}\left(p_{1}\right)^{k-1}+a_{2}\left(p_{1}\right)^{k-2}(p d)^{1}+\cdots+a_{k}(p d)^{k-1}\right) \\
& \geq \sigma(d)=\sigma(f) .
\end{aligned}
$$

This completes the proof of Lemma 2.5.

\section{Proofs of Theorem 1.5}

From Lemma 2.2 we see that there exists a sufficiently large $R$, a positive number $\sigma_{1}\left(\sigma<\sigma_{1}<1\right)$ such that

$$
T\left(32 R, f^{\prime}\right)<R^{\sigma_{1}}
$$

and there exists a set $J_{R} \subseteq[R / 2, R]$ with linear measure $(1-o(1)) R / 2$, such that for any $r \in J_{R},|z|=r$, we have the following equation

$$
H_{k}(f)=\prod_{i=1}^{k} f\left(z+c_{i}\right)-f^{k}(z)=F(z)(1+o(1)),(
$$

where $F(z)$ satisfies the following express,

$$
F(z)=f^{\prime}(z) \phi(z),
$$

here $\phi(z)=\sum_{m=11 \leq j_{1} \leq j_{2} \leq \cdots \leq k} \sum_{j_{1}} c_{j_{2}} \cdots c_{c_{j_{m}}}\left(f^{\prime}\right)^{m-1} f^{k-m}$.

On the other hand, under the condition of Theorem 1.5 and from Lemma 2.4 we know $H_{k}(f)$ transcendental.

Suppose that $\varepsilon$-set $\mathrm{E}$ concludes all of zeros and poles of $H_{k}(f), f(z), f\left(z+c_{1}\right), \cdots, f\left(z+c_{k}\right)$, and $f^{\prime}(z)$. Setting

$$
\begin{aligned}
& E_{R}=\{r: r \in E,|z|=r<R\}, \\
& E_{\infty}=\{r: r \in E,|z|=r<\infty\} .
\end{aligned}
$$

Since the property of $\varepsilon$-set and $\sigma_{1}<1$, we have that $E_{\infty}$ is with finite logarithmic measure, and $E_{R}$ has linear measure $o(1) R / 2$ for sufficiently large $R$.

We assume that $F_{R}$ is a set, such that

$$
F_{R}=\left\{r: r \in\left[\frac{R}{2}, R\right], n(r, f)=n\left(r-\sum_{j=1}^{k}\left|c_{j}\right|, f\right)\right\} .
$$

Noting that there exists $o(R)$ many points $q_{l} \in\left[\frac{R}{3}, R\right]$ at most from (2.22), at which $n(t, f)$ is not continuous, and also for any $r \in[R / 2, R]$,

$r \in\left[q_{l}, q_{l}+\sum_{j=1}^{k}\left|c_{j}\right|\right]$ holds for some $l$ whenever $n(r, f)>n\left(r-\sum_{j=1}^{k}\left|c_{j}\right|, f\right)$. Therefore, $F_{R}$ has linear measure

$$
m\left(F_{R}\right) \geq(1-o(1) R / 2),
$$

From (2.23)-(2.25), we know that there exists $r \in F_{R} \cap J_{R} \backslash E_{R}$ such that $H_{k}(f), f(z), f\left(z+c_{j}\right)$, $j=1,2, \cdots, k$, and $f^{\prime}(z)$ have no zeros and poles on the circle $|z|=r$. Therefore,

$$
\left|H_{k}(z)-F(z)\right|<|o(1) F(z)|<|F(z)| .
$$

Applying Rouché's Theorem to $H_{k}(f)$ and $F(z)$, we obtain the following equation

$$
n\left(r, \frac{1}{H_{k}(f)}\right)=n\left(r, \frac{1}{F}\right)-n(r, F)+n\left(r, H_{k}(f)\right) \text {. }
$$

Without loss of generality, we may assume that

$$
f\left(z_{0}+\sum_{j=1}^{k} l_{j} c_{j}\right) \neq 0, \infty\left(l_{j}=0, \pm 1, j=1,2, \cdots, k\right)
$$

for all poles $z_{0}$ of $f(z)$. From the assumption in Theorem 1.3, we know that there exists positive number $r_{0}>0$, which does not depend on $R$ and $r$, such that if $z_{0}$ is a pole of $f(z)$ with multiplicity $n$,

$$
r_{0}<\left|z_{0}\right|<r-\sum_{j=1}^{k}\left|c_{j}\right|,
$$

then by the expression of $H_{k}(f)$ and $H_{k}\left(f\left(z-c_{j}\right)\right)$,

$$
H_{k}\left(f\left(z-c_{j}\right)\right)=\sum_{t=1}^{k} f\left(z+c_{t}-c_{j}\right)-f^{k}\left(z-c_{j}\right)
$$

we see that $z_{0}, z_{0}-c_{j}(j=1,2, \cdots, k)$ are respectively the pole of $H_{k}(f)$ with multiplicity $k n, n$. Therefore, we deduce that

$$
n\left(r, H_{k}(f)\right) \geq 2 k n(r, f)+O(1) .
$$

Since the pole $z_{0}$ of $F(z)$ has multiplicity $k(n+1)$, we have the following equality

$$
n(r, F)=k(n(r, f)+\bar{n}(r, f)) .
$$

And obviously,

$$
n\left(r, \frac{1}{F}\right)=n\left(r, \frac{1}{f}\right)+n\left(r, \frac{1}{\phi(f)}\right) .
$$


Substituting (2.28), (2.30) into (2.27), we obtain

$$
\begin{aligned}
& \begin{aligned}
n\left(r, \frac{1}{H_{k}(f)}\right) \geq & n\left(r, \frac{1}{f^{\prime}}\right)+n\left(r, \frac{1}{\phi(f)}\right) \\
& +k[n(r, f)-\bar{n}(r, f)]+O(1),
\end{aligned} \\
& \text { If } \bar{\lambda}\left(\frac{1}{f}\right)<\lambda\left(\frac{1}{f}\right), \text { then } n(r, f)=o(n(r, f)) . \text { Thus, }
\end{aligned}
$$

we have that by (2.31)

$$
n\left(r, \frac{1}{H_{k}(f)}\right) \geq n\left(r, \frac{1}{f^{\prime}}\right)+n(r, f)+O(1),
$$

then $\lambda\left(H_{k}(f)\right)=\sigma\left(H_{k}(f)\right)=\sigma(f)$.

If $\bar{\lambda}\left(\frac{1}{f}\right)=\lambda\left(\frac{1}{f}\right)$, we have that from (2.31)

$$
n\left(r, \frac{1}{H_{k}(f)}\right) \geq n\left(r, \frac{1}{f^{\prime}}\right)+n\left(r, \frac{1}{\phi(f)}\right)+O(1) \text {. }
$$

By Lemma 2.5 and (2.33), we deduce that

$$
\lambda\left(H_{k}(f)\right)=\sigma\left(H_{k}(f)\right)=\sigma(f) .
$$

In particular, if $z_{0}$ is the zero of

$$
\psi_{k}(f)=H_{k}(f) / f^{k}(z),
$$

then $z_{0}$ is, also the zero of $H_{k}(f)$. On the other hand, if $z_{1}$ is the zero of $H_{k}(f)$, but not the zero of $\psi_{k}(f)$, then $z_{1}$ must be the zero of $f(z)$, that is, $f\left(z_{1}+c_{j}\right)=0$ for some $j$. From the assumption in Theorem 1.5 that $f(z)$ has at most finitely many zeros $z_{j}, z_{s}$ satisfying $z_{j}-z_{s}=c_{1}, c_{2}, \cdots, c_{k}$, we have

$$
n\left(r, \frac{1}{\psi(f)}\right)=n\left(r, \frac{1}{H_{k}(f)}\right)+O(1) .
$$

Therefore, $\lambda(\psi(f))=\sigma(\psi(f))=\sigma(f)$.

\section{REFERENCES}

[1] J. M. Whittaker, "Interpolatory Function Theory,” Cambridge Tracts in Mathematics and Mathematical Physics, No. 33, Cambridge University Press, New York, 1935, p. 52.

[2] M. Ablowitz, R. G. Halburd and B. Herbst, "On the Extension of Painleve Property to Difference Equations," Nonlinearty, Vol. 13, No. 3, 2000, pp. 889-905. doi:10.1088/0951-7715/13/3/321

[3] R. G. Halburd and R. Korhonen, "Difference Analogue of the Lemma on the Logarithmic Derivative with Applications to Difference Equations," Journal of Mathematical Analysis and Applications, Vol. 314, No. 2, 2006, pp. 477 487. doi:10.1016/j.jmaa.2005.04.010

[4] R. G. Halburd and R. Korhonen, "Nevanlinna Theory for the Difference Operator,” Annales Academiae Scientiarum Fennicae Mathematica, Vol. 31, No. 2, 2006, pp. 463478.

[5] I. Laine and C. C. Yang, "Value Distribution of Difference Polynomials," Proceedings of the Japan Academy, Vol. 83, No. 8, 2007, pp. 148-151. doi:10.3792/pjaa.83.148

[6] Y. M. Chiang and S. J. Feng, "On the Nevanlinna Characteristic of $f(z+c)$ and Difference Equations in the Complex Plane,” The Ramanujan Journal, Vol. 16, No. 1, 2008, pp. 105-129. doi:10.1007/s11139-007-9101-1

[7] W. Bergweiler and J. K. Langley, "Zeros of Differences of Meromorphic Functions,” Mathematical Proceedings of the Cambridge Philosophical Society, Vol. 142, No. 1, 2007, pp. 133-147. doi:10.1017/S0305004106009777

[8] Z. X. Chen and K. H. Shon, "On Zeros and Fixed Points of Difference of Meromorphic Functions,” Journal of Mathematical Analysis and Applications, Vol. 344, No. 1, 2008, pp. 373-383. doi:10.1016/j.jmaa.2008.02.048

[9] Z. X. Chen and K. H. Shon, "Estimates for the Zeros of Difference of Meromorphic Functions,” Science China, Series A, Vol. 52, No. 11, 2009, pp. 2447-2458. doi:10.1007/s11425-009-0159-7 